\title{
Cholesterol-lowering Potential and Exopolysaccharide Biosynthesis of Lactobacillus spp. isolated from Human Milk
}

\author{
Pham Thi Thu Uyen, Nguyen Hoai An, Pham The Hai, Bui Thi Viet Ha* \\ VNU University of Science, 334 Nguyen Trai, Thanh Xuan, Hanoi, Vietnam
}

Received 19 September 2021

Revised 01 November 2021; Accepted 10 November 2021

\begin{abstract}
Recent research generated information that human milk is not only a valuable source of nutrition, but it also provides a complex microbial community, containing especially Lactobacillus species - the major components of a great number of commercial probiotics. New findings on potential applications of Lactobacillus species revealed that these bacteria have abilities to produce anti-microbial exopolysaccharides (EPS) and to reduce cholesterol in culture broth. In this study, we successfully isolated and screened for Lactobacillus bacteria from human milk samples, and finally obtained four strains, including L. plantarum BM7.13, L. plantarum BM29.7, L. acidophilus BM10.8 and L. rhamnosus BM30.4. Researching the probiotic activities of these strains showed that all strains were tolerant to the low $\mathrm{pH}$ (3.0) and $0.3 \%$ bile salts. Characterization of the probiotic properties indicated that all selected Lactobacillus isolates had ESP $(125-326 \mathrm{mg} / \mathrm{L})$ and exhibited strong antimicrobial activities against pathogenic microbes, such as Escherichia coli, Staphylococcus aureus, Shigella flexneri and Salmonella typhimurium. Our results also indicated that all strains displayed cholesterol assimilation capabilities in culture broth with the maximum figure recorded for L. plantarum BM7.13.
\end{abstract}

Keywords: Lactobacillus, decrease cholesterol, probiotics.

\section{Introduction}

Lactic acid bacteria (LABs) are common microorganisms that play an important role in the human gut microbiome. Due to the ability of producing organic acids, especially lactic acid, this group of bacteria has been widely applied in biological fermentation products. Among the LAB, Lactobacillus is the largest group with over 200 species, which has popular application in commercial probiotics, including

\footnotetext{
* Corresponding author.

E-mail address: buithivietha@hus.edu.vn

https://doi.org/10.25073/2588-1140/vnunst.5329
}

L. acidophilus, L. rhamnosus, L. reuteri, L. casei and $L$. plantarum. Probiotics from Lactobacillus are also employed as an alternative therapy to antibiotics because of their ability to inhibit pathogens $[1,2]$.

One of the valuable health applications of Lactobacillus is antimicrobial activity via the ability to biosynthesize antimicrobial molecules, such as ethanol, fatty acid, hydrogen peroxide, bacteriocins and especially exopolysaccharides (EPS). Reports on LAB-derived EPSs suggested that they exhibited antagonistic role to microbial pathogens. For instant, the EPS of L. casei NA-2 was discovered to antibiofilm formation from Bacillus cereus (95.5\%), 
S. aureus $(30.2 \%)$ and E. coli $(16.9 \%)$ [3]. Recently, research on EPS have been attracted the attention of many scientists around the world, because of its ability to enhance bacteria cooperation with the environment, protect Lactobacillus against the development of harsh conditions, including bile salts, hydrolyzing enzymes, lysozyme, gastric and changes in $\mathrm{pH}$, temperature or osmolarity, to scavenge a broad spectrum of free radicals and have capable of binding free cholesterol [3].

Another applicable health-promoting function of Lactobacillus is the mechanism of lowering serum cholesterol levels, which related to cardiovascular disease (CVD) treatment. According to the World Health Organization (WHO), around $30 \%$ of human deaths globally are attributed to CVD. Therefore, it is hypothesized that if Lactobacillus have capable to reduce excess cholesterol in the intestinal tract, it will have great prospective in preventing CVD [4].

There is an increasing evidence that Lactobacillus species are one of the most dominant bacteria in human milk, making human milk is not only the first source of nutrition for infants, but also contains beneficial bacterial that undoubtedly contributed to human well-being protection [5]. In our previous work $[1,2]$, we successfully isolated $L$. reuteri SMH02 and $L$. gasseri SMH15 from Vietnamese human milk, in which the former was produced commercially as Lacvagin probiotics to strengthen vaginal health and the latter was reported to have a capability to cholesterol assimilate.

Following previous research, in this report, we present our novel finding in the potential health-promoting functions of EPS and assessment the cholesterol reduction of newly isolated strains of Lactobacillus from human milk.

\section{Materials and Methods}

\subsection{Bacterial Isolation}

The human milk samples were collected from 40 healthy women in Northern Vietnam between May 2020 and October 2020. The participating mothers acknowledged to sign a consent form and avoid intake of antibiotics or any food supplements containing added lactic acid bacteria within 2 weeks prior to the collection day [6]. Milk samples were collected in sterile tubes and stored in a laboratory freezer at minus $20{ }^{\circ} \mathrm{C}$ until further processing. The project was approved by the Ethical Committee under approval number IRB-1906.

Collected human milk samples were unfrozen in the refrigerator overnight and left at room temperature for $30 \mathrm{~min}$ before going to bacterial inoculation step. Aliquots of $100 \mu \mathrm{l}$ of 10 fold milk dilution in $0.15 \mathrm{M} \mathrm{NaCl}$ were directly plated on de Man Rogosa Sharpe (MRS - a specific medium for lactobacilli) agar plates and incubated for $48 \mathrm{~h}$ at $37{ }^{\circ} \mathrm{C}$ under anaerobic conditions [2].

DNA extraction from bacterial colonies was performed using ANAPURE DNA mini kit (Anabio, Vietnam). The identification of Lactobacillus spp. was analyzed based on the sequence of $16 \mathrm{~S}$ rDNA with PCR reactions to amplify $1500 \mathrm{bp}$ fragments using forward primer 63F (5'- GCGGCGTGCCTAATACATGC -3') and reverse primer 1378R (5'- AAGGCCCGGG AACG -3'). A typical PCR mix $(25 \mu \mathrm{l})$ consisted of $2 \mathrm{X}$ OneTaq $^{\circledR}$ DNA Polymerase (New England Biolabs, USA), $0.5 \mu \mathrm{M}$ primers and $2 \mu \mathrm{l}$ DNA. Thermocycler incubation using Mastercycler $^{\circledR}$ Nexus-PCR Thermal Cycler (Eppendorf, Germany) followed general conditions: $94{ }^{\circ} \mathrm{C}$ for $2 \mathrm{~min} ; 35$ cycles at $94{ }^{\circ} \mathrm{C}$ for $40 \mathrm{~s}, 60^{\circ} \mathrm{C}$ for $45 \mathrm{~s}, 68^{\circ} \mathrm{C}$ for $90 \mathrm{~s} ; 1$ cycle at $68{ }^{\circ} \mathrm{C}$ for 5 mins and hold at $4{ }^{\circ} \mathrm{C}$. The integrity of the PCR products was performed by acquiring 1500 bp DNA bands followed electrophoresis for 45 mins at $100 \mathrm{~V}$ in $1 \%$ $(\mathrm{w} / \mathrm{v})$ agarose gels in TAE buffer. DNA sequencing was undertaken by the Institute of DNA Technology and Genetic Analysis (GENLAB, Vietnam). Sequences were analyzed using Nucleotide Basic Local Alignment Search Tool (BLAST) (National Center for Biotechnology Information, USA) (https://blast.ncbi.nlm.nih.gov/Blast.cgi). 


\subsection{Titration Acidity}

Titratable acidity (TA) was determined by the standard titration procedure for total titratable acidity (TTA) according to A.O.A.C, (1990) [7]. MRS bacteria broth was fermented for $40 \mathrm{~h}$ at $37^{\circ} \mathrm{C}$ under anaerobic conditions. Lactic acid analysis was performed at 2, 5, 12, $14,17,19,24,36$ and $40 \mathrm{~h}$ by titrating $10 \mathrm{~mL}$ of the supernatant fluid of the substrates on addition of 1 drop phenolphthalein as indicator, neutralized by adding slowly $0.1 \mathrm{M}$ Sodium hydroxide $(\mathrm{NaOH})$ (until a pink colour appeared). Each $\mathrm{mL}$ of $0.1 \mathrm{M} \mathrm{NaOH}$ is equivalent to $90.08 \mathrm{mg}$ of lactic acid. Lactic acid $(\mathrm{mg} / \mathrm{mL})$ was calculated using the following equation: TA $(\mathrm{g} / 100 \mathrm{~mL})=(\mathrm{V} \mathrm{NaOH} \times \mathrm{N} \mathrm{NaOH} \times 90.08)$ V sample.

\subsection{Acid Tolerance}

Aliquots $(100 \mu \mathrm{l})$ of overnight bacteria cultures were inoculated into $10 \mathrm{~mL}$ MRS broth with $\mathrm{pH}$ from 2.0 to 6.0 for 3 hours. Acid tolerance was determined by comparing bacterial growth at time $=0\left(\mathrm{~T}_{0}\right)$ and time $=3$ $\left(\mathrm{T}_{3}\right)$ via measuring the absorbance value of cultures in a photometer at $620 \mathrm{~nm}$ [6].

\subsection{Bile Salt Tolerance}

Bacteria cultures were grown in $10 \mathrm{~mL}$ of agitated liquid MRS medium for 24 hours. The following day, the bacteria cultures were supplemented with $10 \%$ filter sterilised bile salts (Sodium salt taurocholic acids, Sigma, USA) to give a final concentration of $0.3 \%$ bile salts for culture broth. Bile salt tolerance of the bacteria strains was analysed via bacterial population at $\mathrm{T}_{0}$ and $\mathrm{T}_{4}$ with optical density of the cultures measured at a wavelength of $620 \mathrm{~nm}$ [6].

\subsection{Cholesterol Removal}

Cholesterol removal ability of the growing, resting, and dead cell lactic acid bacterial strains was measured following the method described by Anila et al. [8]. Each lactic acid bacterial strain was grown overnight in three 10 mL MRS broth flasks namely R1, D1, G1. The following day, cell pellets were harvested separately from R1 and D1 cultures by centrifuging at $10,000 \mathrm{rpm}$ at $4{ }^{\circ} \mathrm{C}$ for $15 \mathrm{~min}$ and washed twice with sterile distilled water. The resting cells in $\mathrm{R} 1$ tubes were re-suspended in $10 \mathrm{~mL}$ of sterile $0.05 \mathrm{M}$ phosphate buffer ( $\mathrm{pH}$ 6.2) containing $0.3 \%$ bile salts and $100 \mathrm{mg} / \mathrm{mL}$ water-soluble cholesterol (Sigma, USA). For preparation of heat-killed cells, the cell pellets in D1 tubes were re-suspended in $10 \mathrm{~mL}$ of sterile distilled water and autoclaved for $15 \mathrm{~min}$ at $121{ }^{\circ} \mathrm{C}$. The dead cells were centrifuged at $10,000 \mathrm{rpm}$ at $4^{\circ} \mathrm{C}$ for $15 \mathrm{~min}$ and re-suspended in $10 \mathrm{~mL}$ of MRS broth containing $0.3 \%$ bile salts and $100 \mathrm{mg} / \mathrm{mL}$ water-soluble cholesterol. The growing lactic acid bacterial strains were performed by transferring 2\% (v/v) G1 overnight cultures to freshly prepared MRS broth containing $100 \mathrm{mg} / \mathrm{mL}$ water-soluble cholesterol and $0.3 \%$ bile salts. All the growing, resting, and dead cell cultures were incubated for $24 \mathrm{~h}$ and $48 \mathrm{~h}$ at $37^{\circ} \mathrm{C}$ under anaerobic conditions.

Cholesterol assimilation by growing, resting, and dead cells was calculated via calorimetric identification of the remaining cholesterol in the cultures after removing bacteria followed the method reported by Alp Avci. The cholesterol analysis was finally achieved using the formula: A $(\%)=100$ $[(\mathrm{B} / \mathrm{C}) \mathrm{x}$ 100] where $\mathrm{A}$ was cholesterol elimination (\%); $\mathrm{B}$ and $\mathrm{C}(\mu \mathrm{g} / \mathrm{mL})$ were cholesterol amount in the inoculated medium and in the control medium, respectively [9].

\subsection{Bile Salt Hydrolase (BSH) Activity Testing}

The BSH activity of isolates was examined applying the method of Anila's group. Overnight cultures were spotted on BSH agar plates (MRS medium supplemented with $0.37 \mathrm{~g} / \mathrm{L} \mathrm{CaCl}_{2}$ and $0.3 \%$ bile salts). The plates were incubated at $37{ }^{\circ} \mathrm{C}$ for $48 \mathrm{~h}$ incubation, and the presence of halos around colonies or a white opaque colony indicated positive BSH activity. Diameters and area of the precipitation zones were analyzed by Fiji software [8].

\subsection{EPS Extraction}

The EPS acquisition and extraction process were performed following Riaz Rajoka's group. 
Aliquots of $100 \mu \mathrm{l}$ overnight bacteria cultures were inoculated into $100 \mathrm{~mL}$ MRS broth and incubated anaerobically at $37{ }^{\circ} \mathrm{C}$ for $48 \mathrm{~h}$. Bacterial cells pelleted at $10 \mathrm{~min}$ at $10,000 \mathrm{rgm}$ and $4{ }^{\circ} \mathrm{C}$ after being treated at $100{ }^{\circ} \mathrm{C}$ for $15 \mathrm{~min}$. The protein in the supernatant was precipitated and removed by $20 \%$ trichloroacetic acid (TCA). Finally, 2V cold ethanol precipitation method was applied to obtain EPS pellets [10].

\subsection{Antimicrobial Activity of EPS}

Antimicrobial activity of EPS was determined by the agar well diffusion method [11]. The pathogent bacteria in this experiment were Escherichia coli ATCC 25922, Staphylococcus aureus ATCC 25923, Shigella flexneri ATCC 12022, Salmonella typhimurium ATCC 14028. Bacteria were cultured overnight at $37{ }^{\circ} \mathrm{C}$ in $\mathrm{LB}$ broth till the concentration reached to $10^{7}-10^{8} \mathrm{CFU} / \mathrm{mL}$. Prior to the experiment, extracted EPS from the four LAB strains were dissolved in deionized water (5 $\mathrm{mg} / \mathrm{mL}$ ) and filter sterilized. Bacterial suspension was spread on LB agar plates according to agar well diffusion method, adding $60 \mu \mathrm{l}$ EPS solution to the well. The plates were incubated at $37^{\circ} \mathrm{C}$ for $24 \mathrm{~h}$. Antimicrobial activity was determined by measuring the diameter of the inhibition zone around the holes.

\subsection{Statistical Analysis}

Statistical significance was calculated using Microsoft Excel software with $\mathrm{p}<0.05$. All experiments were performed in triplicates.

\section{Results and discussion}

\subsection{Isolation and Identification of Lactobacillus Strains from Human Milk}

There are 135 bacterial colonies were obtained from 40 human milk samples, in which four isolates exposed typical characteristics of LAB (Figure 1). Identity of these strains were confirmed by comparing the sequence of the $16 \mathrm{~S} \mathrm{rDNA}$ to sequence databases on NCBI. Results of phylogenetic tree combined with morphological analysis showed that four strains were belonged to Lactobacillus, which were assigned as L. plantarum BM7.13, L. acidophilus BM10.8, L. plantarum BM29.7 and L. rhamnosus BM30.4 (Figure 2). In this study, the species name $L$. plantarum was used instead of Lactiplantibacillus plantarum, as it recently has been re-designated in the new taxonomic notification of IJSEM [12].

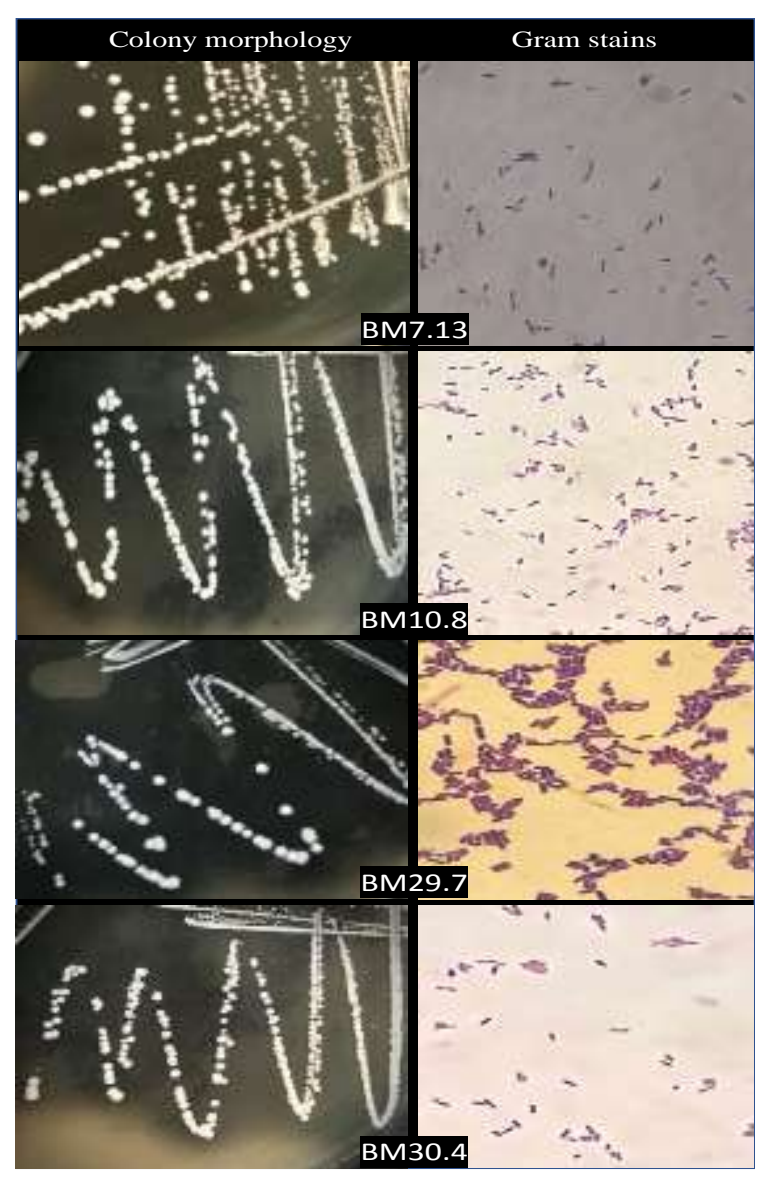

Figure 1. Colony morphology and Gram stains of Lactobacillus strains from human milk after cultured anaerobically on MRS agar at $37^{\circ} \mathrm{C}$ for $48 \mathrm{~h}$.

Research on probiotics in milk suggested that the microbial composition of human milk plays a role in shaping the gut microbiota in breast-fed infants. Common Lactobacillus species constantly present in breast milk were $L$. casei, L. plantarum, L. fermentum, $L$. rhamnosus, and $L$. gasseri, that covers species isolated in this project [13-16]. 


\subsection{Lactic Acid Production and Probiotic Potential}

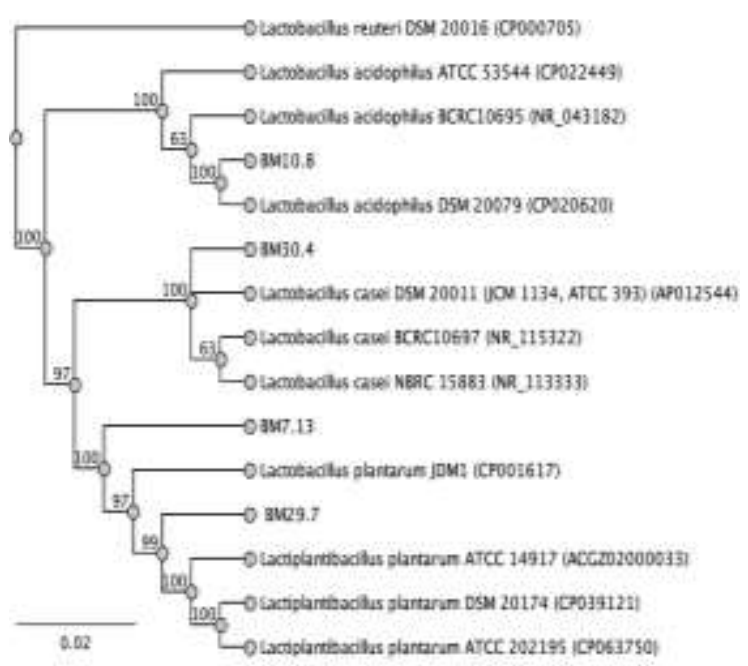

Figure 2. Phylogenetic tree of four isolates based on $16 \mathrm{~S}$ rDNA sequences.

Genetic relatedness of four isolates was constructed by using Geneious Prime 2021 with Genetic Distance Model Tamura-Nei, tree build method Neighbor-joining and Bootstrap value of 1000, using L. reuteri DSM 20016 (GenBank Accession No. CP000705) as the outgroup. LAB have ability to produce lactic acid by transforming the available source of carbohydrates in the media. TA was determined via volume of standard alkali using to neutralize the culture broth. Results of lactic acid production from four isolated LAB in MRS broth are showed in Figure 3. A significantly large amount lactic acid production was obtained from the inoculations. L. acidophilus BM10.8 showed the highest lactic acid production among the four strains $(2.43 \mathrm{~g} / 100 \mathrm{~mL}$ at $36 \mathrm{~h}$ ), followed by L. plantarum BM29.7 $(2.16 \mathrm{~g} / 100 \mathrm{~mL}), \quad$ L. plantarum BM7.13 $(2.16 \mathrm{~g} / 100 \mathrm{~mL})$ and L. rhamnosus BM30.4 $(2.025 \mathrm{~g} / 100 \mathrm{~mL})$ (Figure 3).

The ability to produce lactic acid of the four isolated strains in MRS broth was relatively high compared to the studies of Mis Solval et al., (2019) (1.73 g/100 mL) and Chen et al., (2019) $(28 \mathrm{~g} / \mathrm{L})$ at the same inoculation conditions $[17,18]$.

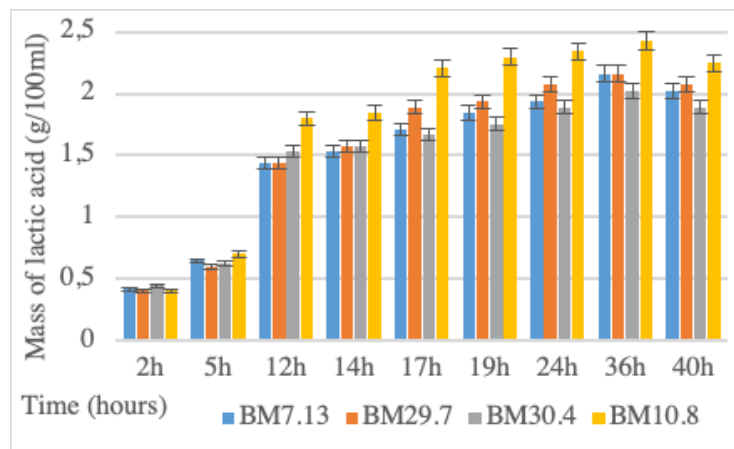

Figure 3. Production of lactic acid by four strains isolates in MRS medium during $40 \mathrm{~h}$ at $37^{\circ} \mathrm{C}$.

The ability to produce lactic acid of the four isolated strains in MRS broth was relatively high compared to the studies of Mis Solval et al., $(1.73 \mathrm{~g} / 100 \mathrm{~mL})$ and Chen et al., (28 g/L) at the same inoculation conditions $[17,18]$.

Lactobacilli employed in fermented foods as probiotics are considered intrinsically resistant to acid environments and bile salt concentration. While approaching the small intestine, they must pass the stressful conditions of stomach. The survival of four Lactobacillus strains, which was examined in acidity conditions $(\mathrm{pH}$ from 2.0 to 6.0 ) and in $0.3 \%$ bile salt medium following the method described by Jiang et al., showed that all four strains were able to survive in $\mathrm{pH} 3.0$ medium and also $0.3 \%$ bile salt medium (data not showed in details) [6].

\subsection{Production of Exopolysaccharide}

There was a considerably great quantity of EPS extraction from the culture broth with the highest number recorded in L. plantarum BM7.13 (326 mg/L), followed by L. acidophilus BM10.8 (316 mg/L), L. rhamnosus BM30.4 (208 mg/L) and finally L. plantarum BM29.7 (125 mg/L). These figures mean that under the same culture conditions, each LAB strain had different ability to synthesize EPS. In particular, L. plantarum BM7.13 could produce EPS content with 2.6 times higher than that produced by $L$. plantarum BM29.7. The yield of EPS from these four strains was much higher than that reported by Dilna' group [19]. 


\subsection{Inhibition of EPS to Pathogens}

Results of the agar well diffusion method (Table 1 and Figure 4) showed that the EPS solution exhibited various degrees of inhibition against tested pathogens with the highest inhibition zone recorded in BM10.8 against S. aureus $(14.6 \mathrm{~mm})$, and $S$. flexneri $(13.1 \mathrm{~mm})$, followed by BM7.13 against E. coli $(12.2 \mathrm{~mm})$ and $S$. typhimurium $(11.1 \mathrm{~mm})$. Compare to the data reported by Riaz Rajoka et al., EPS biosynthesized by $L$. reuteri SHA101 and L. vaginalis SHA110 also had the ability to inhibit pathogenic bacteria, with inhibition zone against S. typhimurium $(15 \mathrm{~mm})$ and E. coli $(13.5 \mathrm{~mm})$ [10].

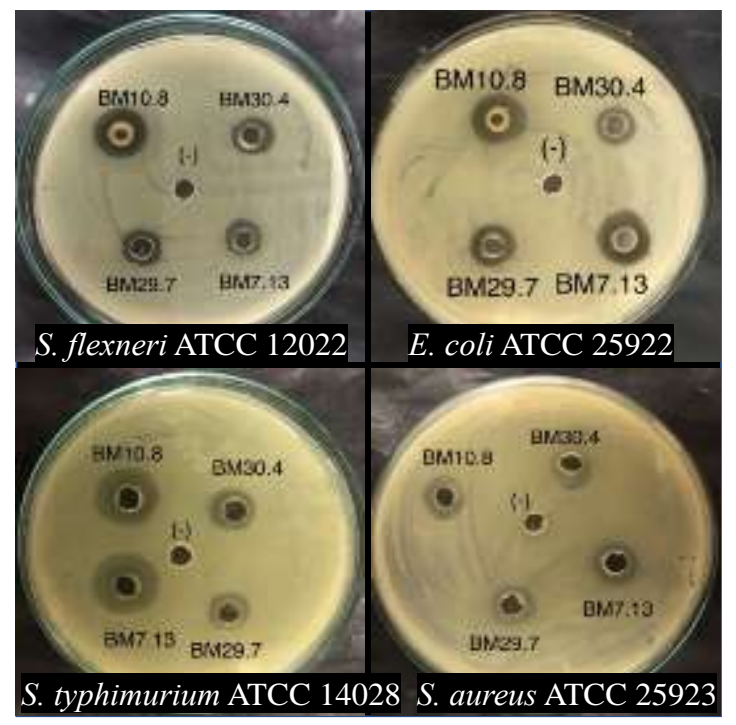

Figure 4. Antimicrobial activity of EPS against pathogenic bacterial species tested.

$S$. typhimurium, $S$. aureus and E. coli are microbial pathogens that cause diseases of the human gastrointestinal tract and spoil food. The antibacterial ability of EPS extraction from Lactobacillus strains opens the novel potential combination of probiotics in the treatment of bacterial infections.

\subsection{Cholesterol Removal}

Levels of cholesterol assimilation during $24 \mathrm{~h}$ and $48 \mathrm{~h}$ of the growing, resting, and dead cell lactic acid bacterial strains are presented in Figure 5. All of four isolated Lactobacillus strains had ability to decrease cholesterol concentration in culture broth. Cholesterol removal varied among strains $(\mathrm{p}<0.05)$ and ranged from $25-75 \%$. Cholesterol assimilation by strains of $L$. plantarum BM7.13 was significantly higher than that of other strains $(\mathrm{p}<0.05)$.

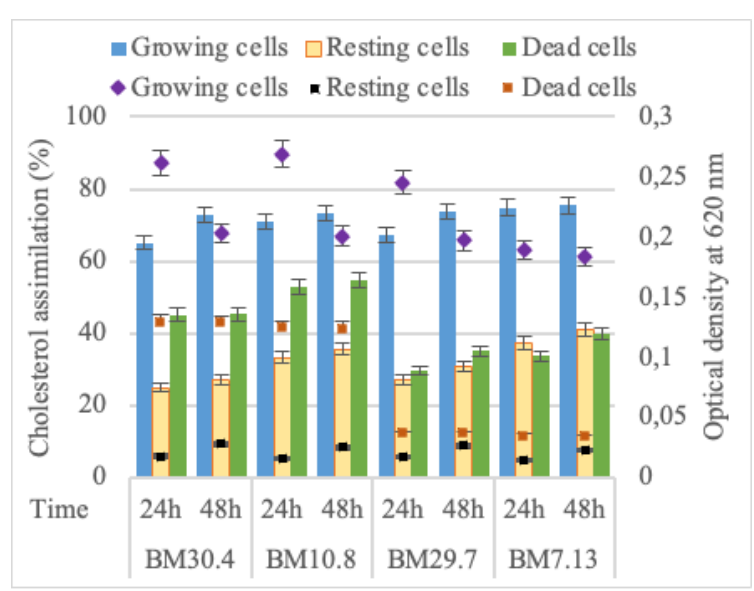

Figure 5. Cholesterol assimilation by studied lactobacilli during $24 \mathrm{~h}$ and $48 \mathrm{~h}$ at $37^{\circ} \mathrm{C}$.

Regarding to the living cell cultures, cholesterol was removed by more than $65 \%$ after $24 \mathrm{~h}$ inoculation, whereas this figure slow down in further $24 \mathrm{~h}$ incubation. Although cholesterol removal capability in this study increased slightly $(\mathrm{p}<0.05)$ as the incubation time increased, the figures showed consistent with cholesterol assimilation patterns in other research [20], indicating that cholesterol removal is growth-associated. Among LAB strains capable of cholesterol assimilation, L. plantarum was reported to demonstrate highest activity, which was compatible with similar research recently [21, 22].

Cholesterol removal rates varied significantly $(p<0.05)$ amongst dead and resting cells, ranging from $30-54 \%$ and $27-41 \%$, respectively. Remarkably, dead cells of BM30.4, BM10.8 and resting cells of BM7.13 were drastically more efficient in cholesterol removal than other strains $(p<0.05)$, meaning that the strain and the cell type influenced cholesterol removal ability expressively. The figures of cholesterol elimination in the resting and dead cells illustrated that their cellular 
membrane had ability to bind cholesterol. Accordingly, it is assumed that even non-viable cells of probiotics have potential application in cholesterol treatments [23]. This result is also consistent with research conducted by Kimoto's group as well as Liong and Shah, that resting and dead cells of Lactobacillus species have capability to remove cholesterol [20, 24]. These figures suggest that cholesterol removal is not only assimilation mechanism of living cells, but also adhesion procedure of cell membranes.

Table 1. Antimicrobial activity of EPS extracted from four Lactobacillus strains

\begin{tabular}{|l|c|c|c|c|}
\hline \multirow{2}{*}{ Strains } & \multicolumn{3}{|c|}{ Inhibition zone (mm \pm SD*) } \\
\cline { 2 - 5 } & $\begin{array}{c}\text { Salmonella } \\
\text { typhimurium }\end{array}$ & $\begin{array}{c}\text { Staphylococcus } \\
\text { aureus }\end{array}$ & Escherichia coli & Shigella flexneri \\
\hline L. plantarum BM7.13 & $11.1 \pm 0.3$ & $13.2 \pm 0.3$ & $12.2 \pm 0.2$ & $10.4 \pm 0.2$ \\
\hline L. acidophilus BM10.8 & $10.9 \pm 0.2$ & $14.6 \pm 0.2$ & $11.4 \pm 0.2$ & $13.1 \pm 0.3$ \\
\hline L. plantarum BM29.7 & $8.1 \pm 0.3$ & $12.5 \pm 0.2$ & $10.5 \pm 0.2$ & $9.6 \pm 0.2$ \\
\hline L. rhamnosus BM30.4 & $9.7 \pm 0.2$ & $11.0 \pm 0.2$ & $9.2 \pm 0.2$ & $9.8 \pm 0.2$ \\
\hline
\end{tabular}

${ }^{*}$ The values are represented as mean $\pm \operatorname{SD}(n=3)$.

\subsection{Bile Salt Hydrolase Activity Testing}

Research on cholesterol metabolism in the human intestinal system has showed that bile salts play an essential role in lipid breakdown and cholesterol absorption. Recently, many strains of Lactobacillus have been discovered owing BSH activity that catalyzes the conversion of conjugated bile salts into free bile salts, showing a potential application in cholesterol-lowering which related to hypercholesterolemia treatment. BSH enzyme gives an advantage to these micro-organisms to survive and colonize the small intestine since the conjugated bile salts have an anti-bacterial effect.

The BSH activity examination of isolates was confirmed by the formation of halos/an opaque precipitation zone formed around the growth of bacterial colonies on the BSH test medium (Figure 6). Three out of four strains produced a large precipitation zone, including both of L. plantarum strains (BM7.13 and BM29.7), then followed by L. acidophilus BM10.8, meaning that these strains possessed extensive BSH activity.

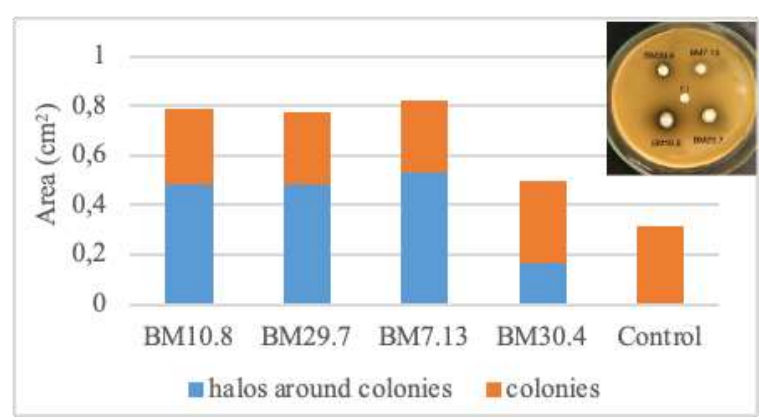

Figure 6. BSH activity of four isolated strains. (The blue indicated the $\mathrm{BSH}$ activity).

The competence of Lactobacillus in BSH activity that contributes to cholesterol-lowering has been reported by great number of studies, in which the strain $L$. plantarum showed a high potential in hydrolyzing bile salts. Research by Yang et al., in 2019 stated that BSH can improve the survival of LAB species in gastrointestinal tract by enhancing their adhesion ability [25]. Wang et al., noticed that $L$. plantarum overexpression of $\mathrm{BSH}$ has beneficial effects against hypercholesterolemia by reducing cholesterol absorption [4]. Recently, Singhal's group remarked the presence of BSH in L. plantarum isolated from 
environment naturally deficient of bile salts and might have a greater adhesion capability for Caco-2 intestinal cell lines [21].

\section{Conclusion}

Our findings in this report provided valuable knowledge that human milk contains a beneficial source of Lactobacillus probiotics, thereby widely opening a prospective application in human well-being protection. There are four Lactobacillus strains with noble potential use were isolated from forty human milk samples. Based on morphological and molecular characteristics, these strains were identified as L. plantarum BM7.13, L. acidophilus BM10.8, L. plantarum BM29.7 and L. rhamnosus BM30.4. Evaluation the probiotic properties of these Lactobacillus strains revealed their ability to produce EPS, which can exert inhibition to pathogens in the experiments. The research also discovered that L. plantarum BM7.13 possessed the highest level of cholesterol-lowering properties and BSH activity, showing that this strain might have a high potential for use as probiotics in supporting the prevention of CVD.

\section{Acknowledgements}

This study was financially supported by Project No. 12.19/CNSHCB of Ministry of Industry and Trade.

\section{References}

[1] T. D. Phan, T. T. H. Tran, T. D. L. Mai, T. H. Pham, T. V. A. Nguyen, T. V. H. Bui, Probiotic Properties of Lactobacillus Gasseri Strain SMH15 Isolated from Breast Milk, Vietnam Trade Ind, Rev, Vol. 12, No. 5, 2020, pp. 382-388 (in Vietnamese).

[2] T. H. Nguyen, T. V. A. Nguyen, T. V. H. Bui, Probiotic Properties of Lactobacillus reuteri SMH2 Isolated from Women Breast Milk, The National Conference on Biotechnology of Vietnam, 2019, pp. 349-355 (in Vietnamese).

[3] A. K. Abdalla et al., Exopolysaccharides as Antimicrobial Agents: Mechanism and Spectrum of
Activity, Front, Microbiol, Vol. 12, No. 5, 2021, pp. 1-15.

[4] G. Wang, W. Huang, Y. Xia, Z. Xiong, L. Ai, Cholesterol-lowering Potentials of Lactobacillus Strain Overexpression of Bile Salt Hydrolase on High Cholesterol Diet-induced Hypercholesterolemic Mice, Food Funct, Vol. 10, No. 3, 2019, pp. 1684-1695.

[5] W. Liu et al., Characterization of Potentially Probiotic Lactic Acid Bacteria and Bifidobacteria Isolated from Human Colostrum, J. Dairy Sci, Vol. 103, No. 5, 2020, pp. 4013-4025.

[6] M. Jiang et al., Evaluation of Probiotic Properties of Lactobacillus plantarum WLPL04 Isolated from Human Breast Milk Breast Milk, J. Dairy Sci, Vol. 99, No. 3, 2016, pp. 1736-1746.

[7] AOAC, Association of Official Analytical Chemists, Official Methods of Analysis, AOAC Arlington, VA, 1990.

[8] K. Anila, A. Kunzes, T. C. Bhalla, In Vitro Cholesterol Assimilation and Functional Enzymatic Activities of Putative Probiotic Lactobacillus sp. Isolated from Fermented Foods/beverages of North West India, J. Nutr Food Sci, Vol. 6, No. 2, 2016, pp. 1-5.

[9] G. Alp Avci, Selection of Superior Bifidobacteria in the Presence of Rotavirus, Brazilian J. Med, Biol, Res, Vol. 49, No. 11, 2016, pp. 1-8.

[10] M. S. Riaz Rajoka et al, Functional Characterization and Biotechnological Potential of Exopolysaccharide Produced by Lactobacillus rhamnosus Strains Isolated from Human Breast Milk, LWT - Food Sci, Technol, Vol. 89, No. 1, 2018, pp. 638-647.

[11] C. Fontana, P. S. Cocconcelli, G. Vignolo, L. Saavedra, Occurrence of Antilisterial Structural Bacteriocins Genes in Meat Borne Lactic Acid Bacteria, Food Control, Vol. 47, No. 1, 2015, pp. 53-59.

[12] A. Oren, G. M. Garrity, Notification that New Names of Prokaryotes, New Combinations, and New Taxonomic Opinions have Appeared in Volume 70, Part 4 of the IJSEM, Int, J. Syst, Evol, Microbiol, Vol. 70, No. 7, 2020, pp. 4050-4060.

[13] C. Jamyuang, P. Phoonlapdacha, N. Chongviriyaphan, W. Chanput, S. Nitisinprasert, M. Nakphaichit, Characterization and Probiotic Properties of Lactobacilli from Human Breast Milk, 3 Biotech, Vol. 9, No. 11, 2019, pp. 1-11.

[14] S. Oddi et al., Occurrence of Bacteria with Technological and Probiotic Potential in Argentinian Human Breast-milk, Benef, Microbes, Vol. 11, No. 7, 2020, pp. 685-702.

[15] C. Luz et al., Probiotic Characterization of Lactobacillus Strains Isolated from Breast Milk and 
Employment for the Elaboration of a Fermented Milk Product, J. Funct, Foods, Vol. 84, No. 7, 2021, pp. 1-9.

[16] D. Ozgun, H. C. Vural, Identification of Lactobacillus Strains Isolated from Faecal Specimens of Babies and Human Milk Colostrum by API 50 CHL System, J. Med, Genet, Genomics, Vol. 3, No. 3, 2011, pp. 46-49.

[17] C. C. Chen et al., Repeated-batch Lactic Acid Fermentation using a Novel Bacterial Immobilization Technique Based on a Microtube Array Membrane, Process Biochem, Vol. 87, No. 2, 2019, pp. 25-32.

[18] K. Mis Solval, A. Chouljenko, A. Chotiko, S. Sathivel, Growth Kinetics and Lactic Acid Production of Lactobacillus plantarum NRRL B-4496, L. acidophilus NRRL B-4495, and L. reuteri B-14171 in Media Containing Egg White Hydrolysates, Lwt, Vol. 105, No. 5, 2019, pp. 393-399.

[19] S. V. Dilna et al., Characterization of an Exopolysaccharide with Potential Health-benefit Properties from a Probiotic Lactobacillus plantarum RJF4, LWT - Food Sci, Technol, Vol. 64, No. 2, 2015, pp. 1179-1186.
[20] M. T. Liong, N. P. Shah, Acid and Bile Tolerance and Cholesterol Removal Ability of Lactobacilli Strains, J. Dairy Sci, Vol. 88, No. 1, 2005, pp. 55-66.

[21] N. Singhal et al., Rhizospheric Lactobacillus plantarum (Lactiplantibacillus plantarum) Strains Exhibit Bile Salt Hydrolysis, Hypocholestrolemic and Probiotic Capabilities in Vitro, Sci, Rep, Vol. 11, No. 1, 2021, pp. 1-9.

[22] R. A. Walhe et al., Cholesterol Reduction and Vitamin B12 Production Study on Enterococcus Faecium and Lactobacillus pentosus Isolated from Yoghurt, Sustain, Vol. 13, No. 11, 2021, pp. 1-11.

[23] E. Tok, B. Aslim, Cholesterol Removal by some Lactic Acid Bacteria that can be used as Probiotic, Microbiology and Immunology, Vol. 54, No. 5, 2010, pp. 257-264.

[24] H. Kimoto, S. Ohmomo, T. Okamoto, Cholesterol Removal from Media by Lactococci, J. Dairy Sci, Vol. 85, No. 12, 2002, pp. 3182-3188.

[25] Y. Yang, Y. Liu, S. Zhou, L. Huang, Y. Chen, H. Huan, Bile Salt Hydrolase can Improve Lactobacillus plantarum Survival in Gastrointestinal Tract by Enhancing their Adhesion Ability, FEMS Microbiol, Lett, Vol. 366, No. 8, 2019, pp. 1-8 